\title{
Topology, Phase Transitions, and the Spherical Model
}

\author{
Sebastián Risau-Gusman, ${ }^{* \dagger}$ Ana C. Ribeiro-Teixeira, ${ }^{\ddagger}$ and Daniel A. Stariolo ${ }^{\S}$ \\ Departamento de Física, Universidade Federal do Rio Grande do Sul, CP 15051, 91501-970, Porto Alegre, Brazil
}

(Received 17 February 2005; published 29 September 2005)

\begin{abstract}
The topological hypothesis states that phase transitions should be related to changes in the topology of configuration space. The necessity of such changes has already been demonstrated. We characterize exactly the topology of the configuration space of the short range Berlin-Kac spherical model, for spins lying in hypercubic lattices of dimension $d$. We find a continuum of changes in the topology and also a finite number of discontinuities in some topological functions. We show, however, that these discontinuities do not coincide with the phase transitions which happen for $d \geq 3$, and conversely, that no topological discontinuity can be associated with them. This is the first short range, confining potential for which the existence of special topological changes are shown not to be sufficient to infer the occurrence of a phase transition.
\end{abstract}

DOI: 10.1103/PhysRevLett.95.145702

PACS numbers: 64.60.- i, 02.40.Sf, 05.70.Fh

Phase transitions (PTs) remain one of the most intriguing and interesting phenomena in physics. Mathematically, a PT is signaled by the loss of analyticity of some thermodynamic function [1] in the thermodynamic limit.

Recently, a new characterization of PTs has been proposed, that conjectures that "at their deepest level PTs of a system are due to a change of the topology of suitable submanifolds in its configuration space" [2]. This is known as the topological hypothesis (TH) [3]. In this new method one studies the topology of the configuration space $\Gamma$ of the potential energy $V(\mathbf{x})$ of a system with $N$ degrees of freedom, determining the changes that take place in the manifolds $M_{v}=\{\mathbf{x} \in \Gamma: V(\mathbf{x}) / N<v\}$ as the parameter $v$ is increased. A topological transition (TT) is said to take place at $c$ if $M_{c-\epsilon}$ and $M_{c+\epsilon}$ are not homeomorphic. The idea is that somehow TTs may be related to PTs.

The necessity of TTs at a phase transition point has been demonstrated for short ranged, confining models [4]. In the $X Y$ model [2] TTs are found both in the mean field (MF) and unidimensional short range versions, whereas a PT is present only in the MF case. This led to a refinement of the TH: only sufficiently "strong" TTs would be able to induce a PT. It was found that in the MF version a macroscopic change of the Euler characteristic happens at exactly the same point $v_{c}$ where a PT appears. Several other models seem to be in agreement with this behavior [5]. But recently it was proved for a nonconfining potential that no topological criterion seems to be sufficient to induce a PT [6]. We show below that the same happens for the spherical model, which is a confining, short ranged potential.

In the spherical model it has been found that there is a direct correlation between the TT and the PT, in its mean field version [7]. Interestingly, in the case of nonvanishing external field there is no PT, but the configuration space displays a TT at energies that cannot be thermodynamically reached.

In this Letter we study the original Berlin-Kac spherical model [8] for spins placed on a $d$-dimensional lattice, interacting with their first neighbors. Using tools from topology theory we were able, for the case of vanishing field, to determine its topology exactly (up to homology). We show that the PT occurring for $d \geq 3$ cannot be related to any discontinuity in the homology of the manifolds at $v_{c}$. For nonvanishing field we cannot characterize the topology completely for all $v$, but show that a very abrupt change in the topology happens that does not have a corresponding PT. At variance with the MF version, the value of $v$ at which this topological change occurs is thermodynamically accessible.

The spherical model is defined by a set of $N$ spins $\epsilon_{i}$ lying on a $d$-dimensional hypercubic lattice and interacting through the potential $V=-\frac{1}{2} \sum_{\langle i j\rangle} J_{i j} \epsilon_{i} \epsilon_{j}-H \sum_{i} \epsilon_{i}$ where $J_{i j}=J$ gives the strength of the interaction between nearest-neighbor spins $i$ and $j$, and $H$ is an external field. The spin variables are real and constrained to lie on the sphere $\mathbb{S}^{N-1}$ (i.e., $\sum_{i} \epsilon_{i}^{2}=N$ ). Periodic boundary conditions are imposed on the lattice.

In [8] it is shown that, at zero field, a continuous PT appears at a critical temperature $T_{c}(d)$ for $d \geq 3$, which is a strictly increasing function of $d$ (see Table I). On the other hand, no PT is possible in an external field.

As in previous works, the thermodynamic function we use to relate the statistical mechanical and topological approaches is the average potential energy per particle $\langle v\rangle$. Although the specific details of $\langle v\rangle$ depend on $d$, some

TABLE I. Critical temperatures $T_{c}$ and mean potential energies per particle $\left\langle\boldsymbol{v}_{c}\right\rangle$ for hypercubic lattices in $d$ dimensions. Values obtained from analytical expressions in [8].

\begin{tabular}{lrc}
\hline \hline$d$ & $k T_{c} / J$ & $\left\langle v_{c}\right\rangle / J$ \\
\hline 3 & 3.9573 & -1.0216 \\
4 & 6.4537 & -0.7728 \\
5 & 8.6468 & -0.6759 \\
6 & 10.7411 & -0.6283 \\
\hline \hline
\end{tabular}


features are common to all hypercubic lattices: $\langle v\rangle \rightarrow 0$ for $T \rightarrow \infty$ and $\langle v\rangle \rightarrow-d$ (its lower bound) when $T \rightarrow 0$.

In the topological approach one looks for changes in the topology of $M_{v}$ as $v$ is increased. A topological change happens at a certain value $v_{T}$ if the manifolds $M_{v_{T}-\epsilon}$ and $M_{v_{T}+\epsilon}$ are not homeomorphic [9] for arbitrarily small $\epsilon$. To make a connection with statistical mechanics Casetti et al. [3] proposed the nontrivial ansatz that, at the phase transition, $\boldsymbol{v}_{T}$ can be identified with $\left\langle\boldsymbol{v}_{c}\right\rangle$, the thermodynamical average critical potential energy per particle. To study the topology of the configuration space of the spherical model it is most convenient to write the potential using the coordinates $x_{i}$ that diagonalize the interaction matrix through an orthogonal transformation:

$$
V=-\frac{1}{2} \sum_{i=1}^{N} \lambda_{i} x_{i}^{2}-\sqrt{N} x_{1} H
$$

where we set $J=1$, and $\lambda_{i}(i=1, \ldots, N)$ are the eigenvalues of the interaction matrix, ordered from largest to smallest. We define the sets $C_{j}, j=0, \ldots, \hat{N}$, where $\hat{N}+1$ is the number of distinct eigenvalues. $C_{j}$ is the set containing the indices of the eigenvalues that have the $(j+1)$ th largest value. Therefore, $\left|C_{j}\right|$ gives the degeneracy associated with the $(j+1)$ th largest eigenvalue. The FrobeniusPerron theorem ensures that the largest eigenvalue is not degenerated, i.e., $C_{0}=\{1\}$.

The critical points of this potential on the sphere $\Gamma=$ $\mathbb{S}^{N-1}=\left\{\mathbf{x} \in \mathbb{R}^{N}: \sum_{j=1}^{N} x_{j}^{2}=N\right\} \quad$ are found using Lagrange multipliers. Along with the spherical constraint, the critical point equations are:

$$
\begin{gathered}
x_{1}\left(2 \mu+\lambda_{1}\right)+\sqrt{N} H=0, \quad x_{i}\left(2 \mu+\lambda_{i}\right)=0, \\
i=2, \ldots, N,
\end{gathered}
$$

where $\mu$ is the Lagrangian multiplier that results from enforcing the spherical constraint. From these equations and Eq. (1) $\hat{N}+1$ critical values of $v$ are obtained, denoted $v_{k}=-\lambda_{l} / 2$, with $l \in C_{k}$, and $k=0, \ldots, \hat{N}$ (ordered from smallest to largest). Notice that the degeneracy of the eigenvalues causes the corresponding critical points to be, in fact, critical submanifolds. This implies that in the directions tangent to the critical submanifolds the Hessian vanishes, which in turn implies that the potential is not a proper Morse function. Nevertheless, using Bott's extension of Morse theory the Euler characteristic can be found exactly [10]. However, profiting from the symmetries of the spherical model we took a more direct route to study its topology. As we show below, for vanishing external field it is possible to characterize completely the topology of the $M_{v}$, by explicitly giving the values of all the Betti numbers of the manifolds.

For $H=0$ the critical manifolds $\Sigma_{v_{j}}, j=0, \ldots, \hat{N}$, are given by $\Sigma_{v_{j}}=\left\{\mathbf{x} \in \Gamma: \sum_{i \in C_{j}} x_{i}^{2}=N\right\}$ [see Eq. (2)]. These are (hyper)spheres whose dimension is given by the degeneracy of the corresponding eigenvalues. To understand the nature of the topological change that happens at the critical values of $v$ it is necessary to know the topology of the $M_{v}$ for $v$ between two critical values. We show below that in the interval $\left(v_{j}, v_{j+1}\right)$ all the manifolds $M_{v}$ are homotopy equivalent to $\mathbb{S}^{D-1}$, where $D$ is the number of eigenvalues larger than $-2 v_{j}$. In fact we prove that $\mathbb{S}^{D-1}$ is a deformation retract of $M_{v}$, which in turn implies their homotopy equivalence [9].

A submanifold $S \subset M$ is a deformation retract of a manifold $M$ if there exists a series of maps $f^{\nu}: M \rightarrow M$ with $\nu \in[0,1]$, such that $f^{0}=I, f^{1}(M)=S$ and $\left.f^{\nu}\right|_{S}=I$ for all $\nu$. The map when considered as $f: M \times[0,1] \rightarrow M$ must be continuous. Let us take $v \in\left(v_{j}, v_{j+1}\right)$. The deformation retract that takes the manifold $M_{v}$ onto its submanifold $\mathbb{S}^{D-1}=\left\{\mathbf{x} \in M_{v}: \sum_{i=1}^{D} x_{i}^{2}=N\right\}$ is given by $\mathbf{x}(\nu)=\left(f_{1}^{\nu}(\mathbf{x}), \ldots, f_{N}^{\nu}(\mathbf{x})\right)$ with

$$
f_{i}^{\nu}(\mathbf{x})=\left\{\begin{array}{ll}
x_{i} \sqrt{1+\nu \sum_{k=D+1}^{N} x_{k}^{2} / \sum_{k=1}^{D} x_{k}^{2}} & \text { for } i \leq D \\
x_{i} \sqrt{1-\nu} & \text { for } i>D
\end{array} .\right.
$$

This map can easily be shown to be continuous at all points $\mathbf{x} \in M_{v}$. The properties for $\nu=0$ and $\nu=1$ are evidently fulfilled. It is also easy to see that the retraction $f^{\nu}$ does not map any points outside $M_{v}$, since the image points always lie on the sphere $\mathbb{S}^{N-1}$, and their potential energy does not exceed $v$.

Homotopy equivalence implies that the Betti numbers of the $M_{v}$ with $v \in\left(v_{j}, v_{j+1}\right)$ are the same as those of $\mathbb{S}^{D-1}$ : $b_{i}\left(M_{v}\right)=1$ for $i=0$ and $i=D-1$, and $b_{i}\left(M_{v}\right)=0$ otherwise. Thus at each $v_{j}$ a topological transition occurs that changes the topology of the phase space from one homotopy equivalent to $\mathbb{S}^{D-\left|C_{j}\right|-1}$ to one homotopy equivalent to $\mathbb{S}^{D-1}$. In terms of the Betti numbers, each transition changes two of them from 0 to 1 and from 1 to 0 . Thus, at variance with other models, the magnitude of the Betti numbers is not a useful quantity in order to characterize the TT. It is better to look at changes in $D-1$, the highest index of the Betti number that changes at each transition. Furthermore, as we have shown that the manifolds $M_{v}$ are homotopy equivalent to (hyper)spheres, the information about their dimension $D-1$ completely characterizes their topology. Thus $D$ is the relevant quantity to be studied. As shown above, the increase of $D$ at each TT is given by the degeneracy of the corresponding eigenvalue. If the degeneracy $\left|C_{j}\right|$ is $o(N)$, given that $D=\sum_{i=0}^{j}\left|C_{i}\right|$, in the $N \rightarrow \infty$ limit $D$ is equivalent to the $\operatorname{order}$ of the critical manifolds, which is defined as the number of negative eigenvalues of the Hessian $\left(\sum_{i=0}^{j-1}\left|C_{i}\right|\right)$ when restricted to the submanifold normal to the $j$ th critical submanifold. This generalizes to degenerate manifolds the definition of order of a saddle point.

For the spherical model it can be shown that the spectrum of eigenvalues is continuous in the infinite $N$ limit. 
Thus, the set of $\hat{N}+1$ critical energies will be dense in $[-d, d]$, the interval of allowed potential energies. Consequently, the model has a continuum of TTs. In this limit, and considering that $D$ is $O(N)$, it is convenient to introduce a continuous and normalized version of $D$, $d(v)=D / N$, and also a degeneracy density $c(v)$. They are related by $c(v)=\frac{\partial d(v)}{\partial v}$. In the following we search for singularities in these functions or their derivatives which could point to particularly strong TTs.

The spectrum of the adjacency matrix is given by [8]:

$$
\lambda_{\mathbf{p}}=2 \sum_{i=1}^{d} \cos \left(2 \pi p_{i} / N^{1 / d}\right), \quad p_{i}=0, \ldots, N^{1 / d}-1 .
$$

In the $N \rightarrow \infty$ limit, the degeneracy density is

$$
\begin{aligned}
c(v) & =(2 \pi)^{d} \int_{0}^{2 \pi}\left(\Pi_{i=1}^{d} d \omega_{i}\right) \delta(v+\lambda(\omega) / 2) \\
& =\int_{0}^{\infty} \frac{d x}{\pi} \cos (x v)\left(J_{0}(x)\right)^{d}
\end{aligned}
$$

where $\lambda(\omega)=2 \sum_{i=1}^{d} \cos \left(\omega_{i}\right)$. It can be shown [11] that the integral converges uniformly for all values of $d$ and therefore $c(v)$ is a continuous function (see Fig. 1). The derivatives with respect to $v$ can be obtained by performing the derivative inside the integral. But, as this is only valid if the resulting integral converges, this procedure allows us to obtain only the first $\lfloor(d-1) / 2\rfloor$ derivatives. All these derivatives are continuous [11] except for the last, which is discontinuous only at the following points: at odd values of $v$ if $d$ is odd, at even values of $v / 2$ if $d / 2$ is odd and at odd values of $v / 2$ if $d / 2$ is even. But these values are
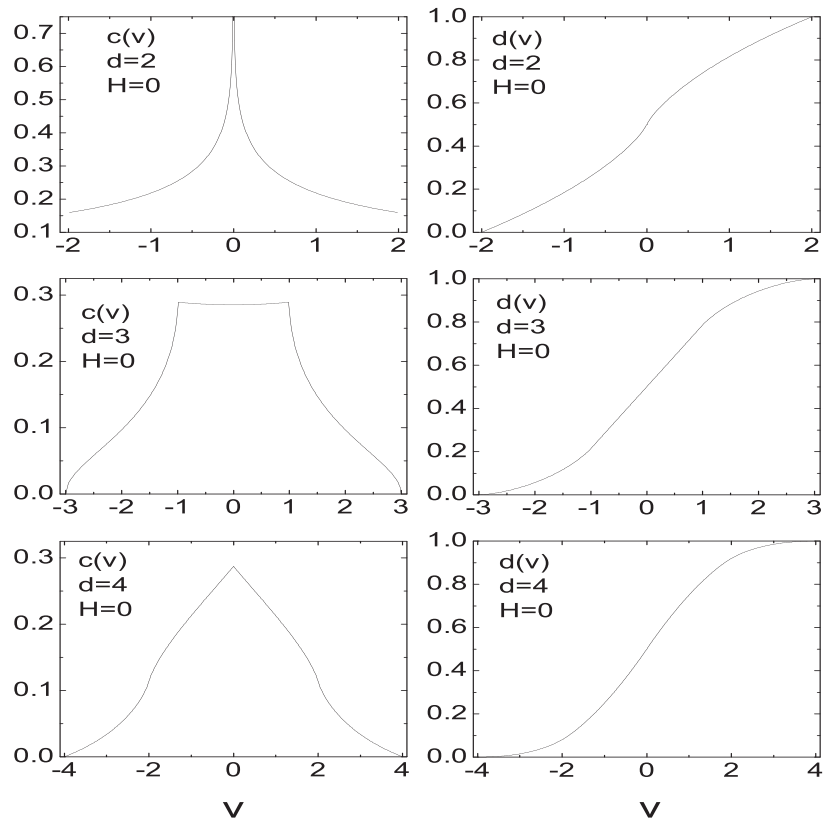

FIG. 1. Degeneracy density $c(v)$ and relative index $d(v)$ of the critical manifolds, for a vanishing external field. clearly different from the ones at which a PT takes place, for all values of $d$ (see Table I). The manifolds $M_{v}$ display TTs at the points $\left\langle v_{c}\right\rangle$ where PTs occur, since there is a continuum of TTs. These TTs, however, are not particularly abrupt. This noncoincidence between the levels where a special TT $\left(\boldsymbol{v}_{T}\right)$ and a PT $\left(\left\langle\boldsymbol{v}_{c}\right\rangle\right)$ take place has also been observed in the $\phi^{4}$ mean field model [12].

The only possibility left to look for a relationship between TT's (in the sense of a discontinuity of some function of the topology) and PT's would be in the higher derivatives of $c(v)$, which cannot be studied by interchanging the integral and derivative operations. This possibility seems to us rather unreasonable, because it would imply not only that the derivative where discontinuities are to be looked for depends on the dimension of the lattice, but also that those discontinuities present in lower order derivatives should be disregarded.

We have thus shown that discontinuities in the derivatives of $c(v)$ are not sufficient to induce the PT present in the model. Furthermore, we show in the following that, even though in the case of a nonzero external field there appear discontinuities in the function $c(v)$ itself, no connection between such TTs and PTs can exist, simply because the model does not display any PTs at all.

With $H \neq 0$ it is not so easy to find the homotopy type of the submanifolds $M_{v}$, because of the breaking of the symmetry introduced by the field term in the Hamiltonian. Nevertheless, using Morse theory it is at least possible to establish the homotopy type of the submanifolds up to above the second smallest critical energy, where an abrupt topological change is shown to take place.

According to Morse theory, if there is one nondegenerate critical point at $c \in(a, b)$, the manifold $M_{c+\epsilon}$ is homeomorphic to $M_{c-\epsilon} \cup e_{k}$, where $e_{k}$ is a $k$ cell (i.e., a $k$-dimensional open disk). In other words, at the critical point, a $k$ cell is attached to the manifold, where $k$ is the index of the critical point, defined as the number of negative eigenvalues of the Hessian at that point.

From the critical point Eq. (2) we obtain that the smallest critical energy is $v_{+}=-\left(\lambda_{1} / 2+H\right)$, and the next is $v_{-}=-\left(\lambda_{1} / 2-H\right)$. The Hessian of the potential on the sphere at the critical points $\mathbf{x}_{ \pm}=( \pm \sqrt{N}, 0, \ldots, 0)$ is a diagonal matrix with $V_{i i}^{ \pm}=\lambda_{1} \pm H-\lambda_{i}(i>1)$. Therefore, at these two points the Hessian is not singular, which implies that $\mathbf{x}_{ \pm}$are nondegenerate critical points. But $\lambda_{1}$ is the largest eigenvalue, therefore for $v_{+}$all the eigenvalues $V_{i i}$ are positive. This was to be expected because this is the absolute minimum of the potential. Topologically this means that for $v<v_{-} M_{v}$ is homotopy equivalent to a disk. For $v=v_{-}$the index of the critical point depends on the field: if $\lambda_{2}<\lambda_{1}-H, v_{-}$is a minimum. Thus, denoting the next critical value by $v_{2}, M_{v}$ for $v \in\left(v_{-}, v_{2}\right)$ is homotopy equivalent to the union of two disjoint disks on the sphere. However, for large values of $N$ the topological scenario is different. Since in this limit the spectrum of the 

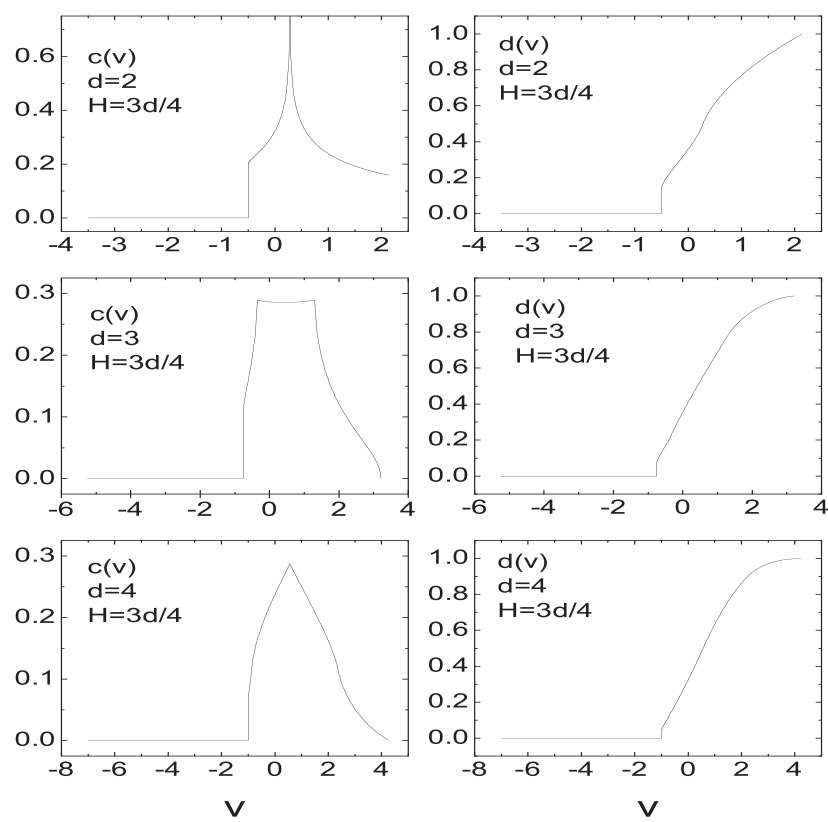

FIG. 2. Degeneracy density $c(v)$ and relative index $d(v)$ of the critical manifolds, for a finite field. A delta function is assumed at the discontinuity point in the graphs of the left column.

adjacency matrix becomes dense, a certain number $k$ of its eigenvalues will fall into the interval $\left(v_{+}, v_{-}\right)$. This number becomes the order of the critical point at $v_{-}$, and gives the dimension of the $k$ cell that is attached to the disk. The manifold $M_{v}$ for $v \in\left(v_{-}, v_{2}\right)$ is therefore homotopy equivalent to a sphere of $k$ dimensions. For large $N, k$ becomes proportional to $N$. In the interval $\left(v_{+}, v_{-}\right)$the manifolds $M_{v}$ have the homotopy type of a point. At $v_{-}$an abrupt change in the topology takes place, and the $M_{v}$ have now the homotopy type of a sphere with a macroscopic number of dimensions [see the jump of $c(v)$ in Fig. 2].

For higher values of $V$, the critical values are given by $v_{j}=-\lambda_{j} / 2+H^{2} / 2\left(\lambda_{1}-\lambda_{j}\right)$, but only for $j$ such that $\lambda_{1}-\lambda_{j}>H$. Notice that, at variance with the case of vanishing $H$, there is a threshold energy below which the critical values have been suppressed. The critical submanifolds occurring at each $v_{j}$ are again hyperspheres whose dimension is given by the degeneracy of the corresponding eigenvalue $\lambda_{j}$. For all critical values we have calculated the order of the critical manifolds, $d(v)$, as well as the relative degeneracy, $c(v)$, for a few values of $d$ (see Fig. 2). The main difference with the results for $H=0$ is that now the connection between the order and the topology of the different manifolds is less obvious, and we have not been able to identify the homotopy types for all values of $v$. Nevertheless we have found exactly the topological change that takes place at $v_{-}$, and have shown that it is macroscopic. It may come as a surprise that this very abrupt change does not have a PT associated with it.
We have shown that the manifolds of the configuration space of the short range spherical model display a continuum of topological transitions. Hence the necessity condition implied by the theorem in [4] is trivially met. Also, strong discontinuities have been found either in a function of the topology or in its derivatives. Although these discontinuities represent abrupt changes in the topology we have shown that they are not associated with PTs. Conversely, at the points where PTs take place no abrupt changes are observed in the topology. These are the first results on a short range confining potential to challenge the sufficiency of a topological mechanism in the origin of a phase transition.

One intriguing question that arises is whether the topologically abrupt changes that we have found can have some influence on the dynamics of the model.

This work was partly supported by $\mathrm{CNPq}$ (Brazil). S. R.-G. acknowledges support from the Centro Latinoamericano de Física. D. A.S. acknowledges support from the Abdus Salam ICTP, Italy.

*Current address: Departamento de Física, CNEA - Centro Atmico Bariloche (R8402AGP) San Carlos de Bariloche, Ro Negro, Argentina.

${ }^{\dagger}$ Electronic address: srisau@cab.cnea.gov.ar

‡Electronic address: anacarol@if.ufrgs.br

${ }^{\S}$ Electronic address: stariolo@if.ufrgs.br

[1] C. N. Yang and T. D. Lee, Phys. Rev. 87, 404 (1952); T. D. Lee and C. N. Yang, Phys. Rev. 87, 410 (1952).

[2] L. Casetti, M. Pettini, and E. G. D. Cohen, J. Stat. Phys. 111, 1091 (2003).

[3] L. Casetti, M. Pettini, and E. G. D. Cohen, Phys. Rep. 337, 237 (2000).

[4] R. Franzosi and M. Pettini, Phys. Rev. Lett. 92, 060601 (2004); R. Franzosi, M. Pettini, and L. Spinelli, math-ph/ 0305032 .

[5] R. Franzosi, M. Pettini, and L. Spinelli, Phys. Rev. Lett. 84, 2774 (2000); L. Angelani, L. Casetti, M. Pettini, G. Ruocco, and F. Zamponi, Europhys. Lett. 62, 775 (2003); P. Grinza and A. Mossa, Phys. Rev. Lett. 92, 158102 (2004).

[6] M. Kastner, Phys. Rev. Lett. 93, 150601 (2004).

[7] A. C. Ribeiro-Teixeira and D. A. Stariolo, Phys. Rev. E 70, 16113 (2004); See, however, an alternative perspective on the problem by M. Kastner, cond-mat/0412199.

[8] T. H. Berlin and M. Kac, Phys. Rev. 86, 821 (1952).

[9] A. Hatcher, Algebraic Topology (Cambridge University Press, Cambridge, 2002).

[10] R. Bott, Bull. Am. Math. Soc. 7, 331 (1982).

[11] S. Risau-Gusman, A.C. Ribeiro-Teixeira, and D. A. Stariolo (to be published).

[12] A. Andronico, L. Angelani, G. Ruocco, and F. Zamponi, Phys. Rev. E 70, 041101 (2004); D. A. Garanin, R. Schilling, and A. Scala, Phys. Rev. E 70, 036125 (2004). 